\title{
Curso online masivo abierto para mejorar la práctica preprofesional en la Universidad Nacional de Educación
}

\author{
Autores: David Olivier Denis Choin \\ Universidad Nacional de Educación, UNAE \\ david.choin@unae.edu.ec \\ Azogues, Ecuador \\ https://orcid.org/0000-0001-8745-2082 \\ Edison Javier Padilla Padilla \\ Universidad Nacional de Educación, UNAE \\ edison.padilla@unae.edu.ec \\ Azogues, Ecuador \\ https://orcid.org/0000-0001-5234-2713
}

\section{Resumen}

Este artículo presenta una experiencia coformadora, cuyo propósito ha sido la mejora de la Práctica Preprofesional a través de la implementación de un curso de formación para tutores internos, externos y estudiantes universitarios, a fin de que todos conozcan sus roles y funciones. Para ello, se han tomado como principales referencias teóricas los aportes de Aguerrondo (1992); Fullan (1998); y Blanco y Messina (2000). Este trabajo sistematiza los elementos innovadores que se congregaron en el Curso Online Masivo y Abierto (MOOC) en la Aproximación al modelo de Práctica Preprofesional. La experiencia de formación, de una duración de 60 horas, se desarrolló en cuatro fases de manera sincrónica y asincrónica. Entre los principales resultados se evidenció la creación de una comunidad de docentesinvestigadores críticos, se generó una motivación basada en la curiosidad del alumnado en un nuevo ambiente de aprendizaje y se organizó una comunidad de aprendizaje interinstitucional. En términos generales, la aplicación del MOOC permitió un cambio de concepción del curso semestral de inducción a la Práctica Preprofesional: desde una perspectiva teórico-presencialmagisterial a una concepción práctica, dialógica y virtual; además, permitió la implementación de estrategias de trabajo autónomas y colaborativas entre los diferentes actores de la Práctica Preprofesional.

Palabras clave: innovación educativa; formación de docentes; formación continua; tutoría; educación.

\footnotetext{
Cómo citar este artículo:

Denis, D., \& Padilla, E. (2021). Curso online masivo abierto para mejorar la práctica preprofesional en la Universidad Nacional de Educación. Revista Scientific, 6(19), 82-101, eISSN: 2542-2987. Recuperado de: https://doi.org/10.29394/Scientific.issn.2542-2987.2021.6.19.4.82101
}

Fecha de Recepción: 23-09-2020
Fecha de Aceptación: 29-12-2020
Fecha de Publicación: 05-02-2021 
OAI-PMH: http://www.indteca.com/ojs/index.php/Revista Scientific/oai

Artículo Original / Original Article

\title{
Open online course to improve pre-professional practice at the National University of Education
}

\begin{abstract}
This article presents a coformative experience, whose purpose has been to improve the Pre-professional Practice through the implementation of a training course for internal and external tutors and university students, so that everyone knows their roles and functions. For this, the contributions of Aguerrondo (1992); have been taken as the main theoretical references; Fullan (1998); and Blanco and Messina (2000). This work systematizes the innovative elements that were gathered in the Massive and Open Online Course (MOOC) in the Approach to the Pre-professional Practice model. The training experience, lasting 60 hours, was developed in four phases, synchronously and asynchronously. Among the main results, the creation of a community of critical teacher-researchers was evidenced, a motivation based on the curiosity of the students was generated in a new learning environment and an interinstitutional learning community was organized. In general terms, the application of the MOOC allowed a change in the conception of the semester induction course to Pre-professional Practice: from a theoretical-classroomteaching perspective to a practical, dialogical and virtual conception; In addition, it allowed the implementation of autonomous and collaborative work strategies between the different actors of the Pre-professional Practice.
\end{abstract}

Keywords: educational innovation; teacher training; training continues; tutoring; education.

\footnotetext{
How to cite this article:

Denis, D., \& Padilla, E. (2021). Open online course to improve pre-professional practice at the National University of Education. Revista Scientific, 6(19), 82-101, e-ISSN: 2542-2987. Recovered from: https://doi.org/10.29394/Scientific.issn.2542-2987.2021.6.19.4.82-101
}

Date Received: 23-09-2020
Date Acceptance:

29-12-2020
Date Publication: 05-02-2021 


\section{Introducción}

El sistema educativo ecuatoriano sufrió varias reformas en los últimos años, en especial en el año 2016, que de acuerdo con Herrera y Cochancela (2020): a fin de asegurar que el sistema educativo ecuatoriano alcance los estándares de calidad educativa, generando una educación equitativa e inclusiva. De esta manera se logrará un desarrollo sostenible para que los ecuatorianos disfruten de una educación de calidad. Paralelamente, y de acuerdo con la Ley Orgánica Reformatoria a la Ley Orgánica de Educación Intercultural del Ministerio de Educación (2015); y la resolución de la Comisión Gestora de la Universidad Nacional de Educación (UNAE, 2015): el Estado emprendió la creación de la Universidad Nacional de Educación del Ecuador, para formar docentes y especialistas altamente calificados que promuevan la innovación y la mejora continua en el sistema educativo.

En este sentido, el énfasis está puesto en la equidad, al asegurar la igualdad de oportunidades de aprendizaje y formación de calidad para los profesionales de la enseñanza que, hasta la fecha en el Ecuador, no dispusieron de los mismos medios que los abogados, médicos, ingenieros 0 arquitectos.

Es así como la UNAE se constituye en una institución de educación superior que innova social y culturalmente, en tanto que asume el nuevo ideal de formación del docente del siglo XXI mediante la implementación de su Modelo Pedagógico, presentado por la Comisión Gestora de la Universidad Nacional de Educación, el cual está fundamentado, tanto en enfoques tradicionales como la sociología y epistemología, como en elementos actuales como la neurociencia y la psicología desde las perspectivas del conectivismo y enactivismo. El objetivo formativo se orienta a la consecución de un triple perfil de salida: docente-investigador-gestor.

La Práctica Preprofesional (PP), conocida en otros ámbitos como formación preparatoria de docentes, prácticum o residencia, ocupa un lugar 
central en el modelo pedagógico de la UNAE, dado que los estudiantes se adentran en el mundo educativo desde el primer hasta el noveno semestre, con un marco curricular definido en torno a tres ejes vertebradores: ayudar, acompañar y experimentar. Así se evita que los practicantes no sepan qué hacer, puesto que la ausencia de planificación y su aislamiento los suman en una situación de abandono, la misma que experimentarán nuevamente al iniciar su carrera docente, una vez graduados. Señalando a Barberi y Pesántez (2017a): para aprovechar todas las oportunidades y los contextos formativos y de desarrollo profesional docente, se creó un modelo de la PP, del que se derivan ejes, roles, funciones de operativización y un sistema de fases de implementación.

Este modelo, al ser de reciente creación, es desconocido por los principales actores que participan en la PP en la UNAE. Cabe precisar que hasta ahora no se realizaba una capacitación formal al modelo de la PP, salvo un conversatorio de tres horas en el que los asistentes no tenían ni voz ni protagonismo. Había una necesidad imperativa de cambio de contexto y de prácticas pedagógicas de formación y capacitación si realmente se deseaba alcanzar los objetivos propuestos: empoderar a los estudiantes y docentes de la UNAE, así como a los tutores de las escuelas, a fin de crear las condiciones idóneas para las PP; comprender la función de la escuela como organismo encargado de la formación práctica de los futuros docentes mediante la gestión de la PP, convirtiendo de esta manera a la práctica en una interacción de beneficio mutuo entre las instituciones de práctica preprofesional y la UNAE.

Sin embargo, para Barberi y Pesántez (2017b): la concepción y el sentido de la Práctica Preprofesional en la UNAE no eran comprendidos por la comunidad educativa: predominaban representaciones, creencias e imaginarios sobre este proceso derivados de las Prácticas Preprofesionales de otras instituciones formadoras de Educación Superior de Cuenca.

Por ello, se consideró necesario un proceso de formación orientado a 
todos los docentes y estudiantes de la UNAE, y los directivos y tutores profesionales (los docentes profesores de los grados en los que los estudiantes de la UNAE desarrollan su PP), dado que es muy difícil llevar a cabo esta capacitación en entornos presenciales. Esto demandaría muchos recursos (tiempo de los actores participantes, docentes facilitadores, y espacios físicos de aprendizaje). Para afrontar esta realidad, se diseñó en la plataforma Moodle un curso en línea, con recursos multimedia de autoría del equipo de gestión de la PP, de carácter introductorio, titulado Aproximación al modelo de Práctica Preprofesional en la UNAE.

La pregunta esencial que generó esta iniciativa innovadora fue: ¿cómo interesar y comprometer a estudiantes, docentes universitarios, directivos y docentes de primaria y secundaria por un modelo de Práctica Preprofesional que supone más trabajo y complicaciones que los anteriores? Para afrontar este reto, el equipo de la PP de la UNAE elaboró un curso virtual que propusiera actividades integradas, contextualizadas a las realidades educativas involucradas, funcionales y que propiciaran la autonomía, participación, comprensión y creatividad de los participantes, a fin de que el factor motivacional garantizara el éxito de la inducción al modelo de la PP, teniendo en cuenta que para la UNAE la innovación educativa es sinónimo de creación de nuevos recursos educativos que permitan desarrollar competencias relacionadas con el Saber, Saber Hacer y Saber Ser.

En las líneas que siguen se presentan los resultados de esta experiencia novedosa en el Ecuador, cuyo objetivo ha sido mejorar la formación inicial docente concientizando a todos los actores de la Práctica Preprofesional de sus roles y funciones en este proceso.

\section{Referentes teóricos y metodológicos}

Innovar es combatir el inmovilismo; es enfrentar los problemas y las deficiencias que se evidencian en las aulas, en la praxis docente y en las 
políticas educativas, sean estas macros, meso o microcurriculares. Así, Cañal (2002a): ha señalado que un centro educativo innovador busca la mejora progresiva del proceso enseñanza-aprendizaje mediante un proceso de evaluación que permite identificar los problemas pedagógicos inherentes a su contexto. Dentro de los procesos evaluativos es común buscar justificativos y responsables externos como administración, disposiciones educativas, contexto, padres de familia, etc. pero la única manera de generar innovaciones es enfrentar sus responsabilidades para mejorar la calidad educativa de su centro de estudios.

Si bien es cierto, existen varias conceptualizaciones de innovación que han sido expresadas por Fullan (1998a); García-Peñalvo (2016); Jiménez, Rappoport, Thoilliez y Navarro (2017); Portilla y Leyva (2018): las cuales suelen equiparar el concepto de innovación educativa a la transformación global de los sistemas educativos. Por ejemplo, Aguerrondo (1992a): hace la distinción entre innovaciones macro (transformación global o reformas estructurales de los sistemas educativos) y micro (cambios parciales o acontecimientos específicos). Las micro, son experiencias de innovación, también conocidas como acontecimientos concretos de innovación.

Nuestra iniciativa, más de allá de la socialización de una práctica pedagógica y formativa exitosa, tiene como finalidad explicitar los conocimientos que, citando a Blanco y Messina (2000a): "subyacen la práctica, recuperarlos, ordenarlos, comunicarlos y traducirlos en propuestas de acción" (pág. 61).

En este orden de ideas, Blanco y Messina (2000b): avisan de la gran relatividad del concepto de innovación en función de "los posicionamientos políticos, sociales, culturales y epistemológicos" (pág. 45); y defienden que la innovación se define de acuerdo con un contexto, una historia, una sociedad y un sistema. Desde luego, lo que ya se implementó ya no puede considerarse novedoso. Por lo tanto, no manejamos el concepto tradicional de innovación 
sino el de cambio de Fullan (1998b); y por extensión, el de Aguerrondo (1992b): que prefiere hablar de acontecimiento concreto de innovación.

Además, nos acogemos a una noción mucho más restringida, pero no menos pertinente ni concreta por ello: las innovaciones por defecto, mencionando a Havelock y Huberman (1980): se generan para dar respuesta a necesidades, carencias y problemas, como un cambio significativo, tanto en el modelo de inducción a la PP como en la responsabilización de sus actores en su rol protagónico de coformadores de futuros docentes. Es entonces, propiamente dicho, una renovación didáctico-pedagógica. Esta renovación se nutre de varios componentes que sustentan su accionar: el ideológico, en relación con el Plan Nacional del Buen Vivir; el cognitivo, alineado con el Modelo Pedagógico UNAE; el ético, acorde al modelo de la PP, y el afectivo, en consonancia con el humanismo.

Según Carbonell (2002a): existen dos enfoques de innovación educativa: el enfoque conservador y el enfoque progresista. El primero se caracteriza por centrarse en la relación, producto-rendimiento, cuyo objetivo prioritario es la competitividad, acentuando así la relación escuela-empresa y acrecentando las desigualdades sociales. En cambio, el segundo enfoque insiste en un proceso colaborativo y diverso que hace énfasis en lo público, al criticar y cuestionar la relación escuela-comunidad y la globalización. Salta a la vista que el enfoque progresista se entronca en la corriente reflexivo-crítica de la educación y en la concepción de la escuela democrática y del aprendizaje a partir de la experiencia de John Dewey (1859-1952). Entonces, nos referimos a una innovación como el pensamiento en la acción.

Las innovaciones educativas exitosas remontan en el mundo occidental a los años 60. En los Estados Unidos, realizaron reformas curriculares de envergadura (especialmente en el área científica relativa a las Matemáticas, la Química y la Física) y, como demuestra Fullan (2002): se debe a las "[...] innovaciones tecnológicas (enseñanza por medio de la TV., máquinas de 
enseñanza), así como innovaciones organizativas (escuelas abiertas, programas flexibles y enseñanza en equipo)" (pág. 2). Por lo tanto, ya hemos pasado la época de las innovaciones (de los años 60 a 90). Al respecto, Fullan (1998c): concluía que la mejora de la calidad educativa estribaba en la aceptación de los usuarios para mejorar sus capacidades, como se aprecia en la figura 1.

Figura 1. El circuito de la mejora de la calidad educativa.

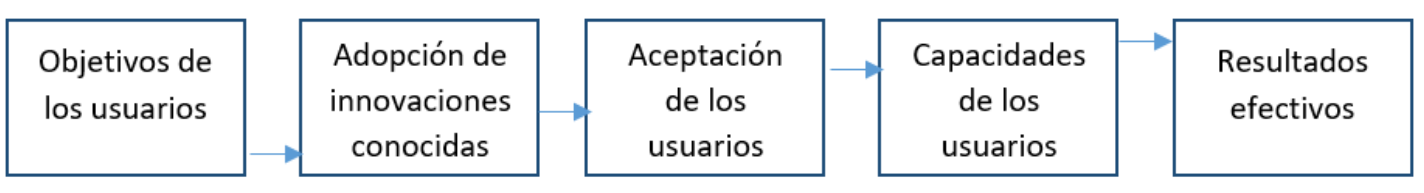

Fuente: Fullan (1998).

En el ámbito latinoamericano la situación es distinta. En los años 60 los que innovaron fueron los técnicos de los ministerios; en los 70 y 80 innovaron los docentes con experiencias innovadoras en la educación formal, no formal y comunitaria. Estas experiencias se fundamentaban en los aportes de corrientes pedagógicas que alzaban la voz en contra del academicismo y de la incapacidad de promover la autonomía, la transformación de la realidad y el pensamiento crítico.

En esa misma línea, Fullan y Pomfret (1977): a finales de la década de los 80 , ya no se referían tanto al concepto de innovación educativa sino al de cambio educacional gracias a la implementación de innovaciones conocidas. En este sentido, Cañal (2002b): señalaba cinco componentes que condicionan el cambio en la medida en que en la práctica se relacionan con "a) materia de estudio o materiales; b) la estructura; c) rol/comportamiento; d) conocimiento y comprensión, y e) internalización de valores" (pág. 6). De ahí que, en el caso de este Curso Online Masivo y Abierto (MOOC), sí se pueda hablar de cambio educacional, ya que se generó un nuevo espacio interinstitucional de formación del profesorado y de construcción conjunta de significados; además, 
cambiaron radicalmente las relaciones entre los miembros de las escuelas de práctica preprofesional y la comunidad educativa UNAE.

\subsection{Estructura y gestión del MOOC}

El MOOC se implementó previo al inicio las prácticas preprofesionales de cada ciclo académico entre octubre del año 2017 y diciembre del año 2020 para socializar y difundir el modelo de la PP de la UNAE a todos los actores involucrados, estudiantes de las carreras de la Universidad, tutores académicos-docentes, tutores profesionales-docentes de las escuelas en las que se desarrolla la PP y directivos de las escuelas de la PP.

La experiencia de formación, de cada implementación del MOOC fue de 60 horas lectivas; estas se desarrollaron en cuatro fases: conocimiento de los roles y funciones; resolución de actividades individuales y grupales en línea; evaluación a través de la respuesta a test y elaboración de planes de actuación; y evaluación de la formación recibida mediante encuestas de opinión.

La estructuración del MOOC se desarrolló con base en los referentes teóricos y metodológicos presentados, dando como resultado la estructuración de un entorno de aprendizaje autónomo que permitió la socialización de los principales elementos de la PP de la UNAE y temáticas afines, mediante un compendio de estrategias, recursos y actividades enfocados a responder los objetivos específicos de cada temática.

EI MOOC tiene por objetivo dar a conocer a todos los actores de la PP, los aspectos más relevantes de la práctica preprofesional. En este se detallan los fundamentos de la PP, se identifican los actores y las actividades a desarrollar en las fases de planeación, investigación y evaluación de las PP. En la tabla 1, se presenta el compendio de los temas, objetivos y actividades que conforman el MOOC. 
Tabla 1. Unidad 1: Los fundamentos del MOOC.

\begin{tabular}{|c|c|c|}
\hline TEMA & OBJETIVO & ACTIVIDADES \\
\hline \multicolumn{3}{|c|}{ Unidad 1. Fundamentos } \\
\hline $\begin{array}{l}\text { Módulo } 1 . \\
\text { La dirección } \\
\text { de la práctica } \\
\text { preprofesional }\end{array}$ & $\begin{array}{l}\text { Identificar las políticas y } \\
\text { normativas educativas que } \\
\text { respalda la actuación de todos } \\
\text { los actores en la PP, } \\
\text { relacionar el modelo de la PP } \\
\text { con el modelo pedagógico de } \\
\text { la UNAE y argumentar los } \\
\text { modos de actuación y } \\
\text { reflexión durante el proceso } \\
\text { formativo de los estudiantes. }\end{array}$ & $\begin{array}{l}\text { Observación del tutorial y realización de } \\
\text { las siguientes actividades: } \\
\text { Lectura de los dos primeros capítulos del } \\
\text { modelo de la PP de la UNAE: 1). Ejes de } \\
\text { fundamentación del modelo; 2). } \\
\text { Concepción de la PP en la UNAE; y } \\
\text { elaboración de un organizador gráfico en } \\
\text { el que se representen los principales } \\
\text { principios y lineamientos. }\end{array}$ \\
\hline
\end{tabular}

Fuente: Los Autores (2020).

\section{Resultados (elementos innovadores)}

Muchos obstáculos materiales, organizativos, logísticos, institucionales e ideológicos ralentizan los procesos educativos entre la universidad y la escuela, y con más razón cuando de alguna innovación, novedad o experimentación se trata. En el caso de este proyecto, innovar es iniciar una dialéctica entre las ideas presentes en el modelo de la PP y la acción que de este se origina. Innovar es cambiar radicalmente las teorías y concepciones de la formación docente en el periodo de la PP, las relaciones de poder entre universidad-escuela, la ausencia de verdadera tutorización de los maestros en ejercicio de los practicantes, sin descuidar el componente axiológico ni la actitud de los estudiantes ante los retos que les presentan las realidades educativas. En síntesis, como señaló Carbonell (2002b): "innovar no es sólo cambiar la práctica docente sino, y sobre todo, cambiar los valores, las creencias y las ideas que fundamentan la acción del profesorado y del alumnado" (pág. 29).

A continuación, se presentan los elementos innovadores de este proyecto de acuerdo con las dos dimensiones del cambio explicadas por Blanco y Messina (2000c): la dimensión objetiva (organización, metodología y 
propósitos) y la subjetiva (participación, compromiso y capacidad crítica de los sujetos sobre la nueva experiencia).

\subsection{Dimensión objetiva}

Uno de los señalamientos comunes formulado a la universidad es su desconexión de la realidad socio-comunitaria, del mundo laboral y, sobre todo, de las escuelas primarias y secundarias. Al respecto, Carbonell (2002c): sentenciaba que "está demostrado que la cultura académica no casa con las otras manifestaciones, expresiones y códigos culturales que se producen más allá de los muros del aula, en otros espacios de socialización y culturización de la infancia y la juventud" (pág. 24). Uno de los principales desafíos del MOOC era lograr la transferencia del modelo -conocimiento e instrumentalización- a las comunidades educativas. La función del Modelo de la PP, y por extensión del MOOC como herramienta para su inducción, es la de planificar, organizar, repensar, sintetizar e integrar dentro de su modus operandi la cultura y el contexto de la escuela, puesto que la PP se realiza en y para la escuela: para conocerla, mejorarla y transformarla.

EI MOOC se estructura en torno a estrategias (vídeos interactivos, organizadores gráficos, cuestionarios, lecturas, etc.) que facilitan la selección, el contraste y el análisis crítico de la información para, en última instancia, transformar a esta en conocimiento procedimental. Así, los participantes comprenden los fundamentos y orígenes del Modelo de la PP, su relación con el modelo pedagógico de la Universidad Nacional de Educación de Ecuador, su instrumentación en la práctica, los roles y las funciones de cada uno de sus actores y, lo que más trascedente es, hacia dónde confluye la coformación de los futuros docentes asumida tanto por la UNAE como por las unidades educativas del Ecuador.

Otro problema recurrente que impide una real transformación de la realidad educativa es la interrupción o abandono de las innovaciones debido a 
la falta de presupuesto o apoyo institucional. En nuestro caso la viabilidad es asegurada, a nivel micro, por el equipo de profesores dedicados semestralmente al seguimiento, monitoreo, sistematización y éxito del MOOC; a nivel mesocurricular, la Dirección de la PP de la Universidad incorpora en el Plan Operativo Anual (POA) un espacio y presupuesto para esta actividad que, recordémoslo, es una piedra angular en el modelo pedagógico de formación docente de la institución.

Asimismo, el MOOC de inducción fue registrado como proyecto de Innovación por la Dirección de Innovación de la UNAE, lo que le permite contar con un presupuesto estimado de 9000 dólares americanos; finalmente, a nivel macrocurricular, la PP, y por extensión el Modelo de la PP y su inducción gracias al MOOC, es respaldada por un convenio ratificado entre la UNAE y el Ministerio de Educación que compromete a las unidades educativas a involucrarse directa y activamente en todo el proceso de Práctica Preprofesional y en sus tres momentos (antes, durante y después). Contamos entonces con las tres condiciones indispensables como indicaba Aguerrondo (1992c): "para que la innovación tenga continuidad y éxito: viabilidad políticocultural; viabilidad organizativa y administrativa y viabilidad material" (pág. 387).

\subsection{Dimensión subjetiva}

En primer lugar, se logró la creación de una comunidad de docentesinvestigadores críticos, puesto que al estar reunidos en la plataforma los estudiantes universitarios, los profesores de la UNAE, los tutores profesionales y directivos de la escuela investigaron, cuestionaron y criticaron su entorno y los procesos de enseñanza-aprendizaje/formación y coformación en esta comunidad educativa variada y múltiple.

En segundo lugar, Usán y Salavera (2018): señalan que la motivación determina el proceso general que guía a la consecución de las metas 
académicas impuestas por los aprendices. En correspondencia con Csikszentmihalyi, Latter y Weinkauff (2017): la motivación en el desarrollo de actividades viene dada por el equilibrio entre los desafíos intelectuales de las actividades propuestas y las capacidades internas para el desarrollo de estas. Es por esa razón que algunas actividades en la plataforma se desarrollaban a través de crucigramas y wikis, lo que permite desacralizar los espacios de aprendizaje y formación al vincularlos con actividades prácticas cotidianas inherentes al ser humano como son la resolución de actividades lógicolingüísticas y la reflexión conjunta. Aflora, por tanto, un clima de motivación, afecto y empatía hacia la Práctica Preprofesional.

En tercer lugar, gracias al MOOC, la universidad y la escuela se transforman en una comunidad de aprendices mutuos según lo enunciaron Cadena-Chala y Orcasitas-García (2016): o en un espacio de interrelaciones mediadoras, que conforme a Vizcarra, Macazaga y Rekalde (2016): afronta el reto de brindar una formación docente significativa, funcional, útil, cooperativa, libre, contextualizada y culturalmente adaptada a las demandas de los sujetos educativos. Estos aprendices se empoderan de su rol gracias a la autonomía y al compromiso que desarrollan en ellos las actividades y el acompañamiento de los tutores. En consecuencia, pasamos de agentes pasivos a agentes activos que contextualizan, piensan, definen y asumen su rol.

En cuarto lugar, hay una ruptura con el individualismo y la formación continua del profesorado. En nuestro caso, todos los docentes ceden sus intereses y protagonismo a los estudiantes. Reflexionan en la acción y en el trabajo colaborativo para alcanzar las condiciones imprescindibles al buen desarrollo de la PP. Los docentes trabajan en parejas pedagógicas, así como los estudiantes UNAE, lo que facilita la compartición de responsabilidades de la formación en la PP con todos sus protagonistas.

En quinto lugar, se genera un clima de confianza y consenso al apuntar hacia proyectos y objetivos comunes. Los estudiantes ayudan a los docentes 
para realizar las actividades del curso y viceversa. Siguiendo Carbonell (2002d): para "crear el ambiente psicológico y ecológico adecuado para la mejora de las relaciones humanas y profesionales" (pág. 18); se trabaja en el aula y en los espacios virtuales (foros y debates). No se debe descuidar el factor humano, esto es, el hecho de que en educación tratamos con seres con experiencias racionales y emocionales anteriores. Por tanto, resulta imprescindible aunar pensamientos, sentimientos y conocimientos; construir un curso interactivo, cercano al usuario, con protagonistas de carne y hueso (no actores profesionales) que, una vez a la semana, interactúan con los participantes en una videoconferencia y, diariamente, orientan a los cursantes por mensajerías.

\section{Conclusiones}

Esta propuesta de inducción al modelo de la PP de la UNAE procura potenciar la relación entre innovación educativa/aplicación innovadora de alguna herramienta y motivación hacia un objeto; en este caso, hacia el conocimiento del Modelo de Práctica Preprofesional de la UNAE y la operativización de los roles de todos sus actores antes, durante y después de la Práctica Preprofesional. Esta reflexión expone experiencias y propuestas de actuación que, quizá, puedan ser replicadas y/o adaptadas en otros contextos y países latinoamericanos.

Este proyecto se propugna otorgar sentido y significado a la PP dentro de la formación docente; otorgar sentido y significado a los roles y las funciones de los actores del modelo de la PP; otorgar protagonismos y legitimidad a las unidades educativas como instituciones coformadoras de los futuros maestros del Ecuador. El desafío, entonces, será alejar por siempre las prácticas rutinarias y consolidar cada vez más este modelo de inducción a la PP que aúna y fomenta el conocimiento, el pensamiento crítico, la praxis pedagógica comprometida y la transformación de los agentes sociales que 
hacen la educación del Ecuador, desde las escuelas primarias y secundarias, pasando por las unidades educativas, hasta llegar a la universidad.

Una vez que se habrá implementado la segunda versión de la inducción a las PP por el MOOC, estaremos en la capacidad de evaluar la adecuación de la innovación y sus impactos a partir de los siguientes interrogantes, que se desglosarán en adelante en varios criterios: ¿ha transformado la situación inicial? ¿Ha resuelto los problemas diagnosticados?; ¿Tiene un impacto educativo (mejor formación del profesorado y educación continua de los docentes en ejercicio) y social (acercamiento universidad-escuela-sociedad)?; ¿Fueron adecuadas las estrategias?; ¿Se resolvieron los conflictos que surgieron antes, durante y después de la inducción?.

Este proceso formativo de aproximación al modelo de la PP tendrá como resultado que cada uno de los actores comprendan los fundamentos y las concepciones que subyacen a los roles que cada uno debe asumir para lograr una formación práctica óptima de los estudiantes de la UNAE. El conocimiento y la comprensión del modelo de la PP son condiciones necesarias para su desarrollo, implementación, evaluación y ajuste. Los resultados de aprendizaje del curso en línea se evidenciarán en la mejora de los procesos y las vivencias en las escuelas de la PP y en la reflexión y teorización en los espacios de aprendizaje en la UNAE.

En resumen, son cuatro los cambios sustantivos logrados gracias a la implementación del MOOC: 1). cambio en la concepción: pasamos de una inducción teórica, presencial, univoca, despersonalizada a una inducción práctica, virtual, democrática y personalizada; 2). cambio en las estrategias: pasamos de estrategias magistricenristas, verbalistas y pasivas, centradas en la transmisión oral de contenidos conceptuales a ofrecimientos de ilustradores, de debate, de aprendizaje colaborativo, modelos, de andamios cognitivos, protagonizados por los cursantes; 3). cambio de estructura: de una simultaneidad de planos y situaciones a una linealidad dividida en tres bloques: 
los fundamentos de la PP, sus actores y Planeación, investigación y teorización de la práctica preprofesional; y 4). cambio en los medios: del papel y de lo audiovisual a lo virtual y digital.

\section{Referencias}

Aguerrondo, I. (1992a,b,c). La innovación educativa en América Latina: balance de cuatro décadas. Perspectivas: Revista trimestral de educación comparada, (3), 381-394, ISSN: 0304-3053. Francia: Organización de las Naciones Unidas para la Educación, la Ciencia y la Cultura - UNESCO.

Barberi, O., \& Pesántez, M. (2017a,b). Experiencia Previa al Modelo de Prácticas de la UNAE. Revista Scientific, 2(3), 53-75, e-ISSN: 25422987. Recuperado de: https://doi.org/10.29394/scientific.issn.25422987.2017.2.3.3.53-75

Blanco, R., \& Messina, G. (2000a,b,c). Estado del arte sobre las innovaciones educativas en América Latina. Colombia: Convenio Andrés Bello; UNESCO.

Cadena-Chala, M., \& Orcasitas-García, J. (2016). Comunidades de aprendizaje en el País Vasco: caracterización y organización escolar. Educación y Educadores, 19(3), 373-391, e-ISSN: 0123-1294. Recuperado de: https://www.redalyc.org/articulo.oa?id=83448566004 Cañal, P. (coord.). (2002a,b). La innovación educativa. ISBN: 84-460-17555. Madrid, España: Ediciones Akal.

Carbonell, J. (2002a,b,c,d). El profesorado y la innovación educativa. En Cañal de León, P. (coord.). La innovación educativa. ISBN: 84-4601755-5. Madrid, España: Ediciones Akal, S.A.

Csikszentmihalyi, M., Latter, P., \& Weinkauff, C. (2017). Running Flow. ISBN: 9781492535720. United States: Human Kinetics.

Fullan, M. (2002). El significado del cambio educativo: Un cuarto de siglo 
de aprendizaje. Profesorado: Revista de curriculum y formación del profesorado, 6(1-2), 1-14, e-ISSN: 1138-414X. Recuperado de: https://dialnet.unirioja.es/servlet/articulo?codigo=2304406

Fullan, M. (1998a,b,c). The Meaning of Educational Change: A Quarter of a Century of Learning. In: Hargreaves A., Lieberman A., Fullan M., Hopkins D. (eds) International Handbook of Educational Change. Kluwer International Handbooks of Education, vol 5, ISBN: 978-94-0106074-5. Dordrecht: Springer.

Fullan, M., \& Pomfret, A. (1977). Research on Curriculum and Instruction Implementation. Review of Educational Research, 47(1), 335-397, eISSN : 0034-6543. Retrieved from:

https://doi.org/10.3102\%2F00346543047002335

García-Peñalvo, F. (2016). En clave de innovación educativa. Construyendo el nuevo ecosistema de aprendizaje. I Congreso Internacional de Tendencias en Innovación Educativa. Arequipa, Perú: CITIE.

Havelock, R., \& Huberman, A. (1980). Innovación y problemas de la educación: Teoría y realidad en los países en desarrollo. ISBN: 923-301527-0. París, Francia: Organización de las Naciones Unidas para la Educación, la Ciencia y la Cultura.

Herrera, M., \& Cochancela, M. (2020). Aportes de las reformas curriculares a la educación obligatoria en el Ecuador. Revista Scientific, 5(15), 362-383, e-ISSN: 2542-2987. Recuperado de:

https://doi.org/10.29394/Scientific.issn.2542-2987.2020.5.15.19.362$\underline{383}$

Jiménez, E., Rappoport, S., Thoilliez, B., \& Navarro, E. (coord.). (2017). Fundamentos de la investigación y la innovación educativa. ISBN: 978-84-16602-55-1. España: UNIR Editorial.

Ministerio de Educación (2015). Ley Orgánica de Educación Intercultural. 
Suplemento del Registro Oficial No. 572 de 25 de agosto. Ecuador: Asamblea Nacional; Dirección Nacional de Normativa Jurídico Educativa.

Portilla, G., \& Leyva, A. (2018). Innovación de la Práctica Docente en la UNAE, Ecuador recurriendo a la Web 2.0. Revista Scientific, 3(7), 140-154, e-ISSN: 2542-2987. Recuperado de:

https://doi.org/10.29394/Scientific.issn.2542-2987.2018.3.7.7.140-154 UNAE (2015). Modelo Pedagógico de la Universidad Nacional del Educación UNAE. Azogues, Ecuador: Universidad Nacional de Educación del Ecuador - UNAE.

Usán, P., \& Salavera, C. (2018). Motivación escolar, inteligencia emocional y rendimiento académico en estudiantes de educación secundaria obligatoria. Actualidades en Psicología, 32(125), 95-112, e-ISSN: 2215-3535. Recuperado de:

http://dx.doi.org/10.15517/ap.v32i125.32123

Vizcarra, M., Macazaga, A., \& Rekalde, I. (2016). ¿Cómo se resuelven los conflictos en tres comunidades de aprendizaje?. Revista de psicodidáctica, 21(2), 281-301, e-ISSN: 1136-1034. Recuperado de: https://dialnet.unirioja.es/servlet/articulo?codigo $=5565741$ 


\section{David Olivier Denis Choin \\ e-mail: david.choin@unae.edu.ec}

Nacido en Saint Quentin, Francia, el 10 de noviembre del

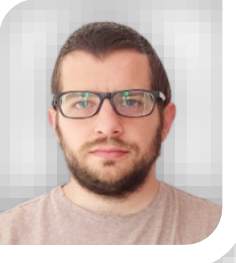
año 1987. Licenciado en Estudios Culturales Hispánicos por la Université de Picardie Jules Verne d'Amiens (UPJV), Francia; y en Filología Hispánica por la Universidad de Alicante (UA), España; Máster en Estudios Literarios y Doctor en Filosofía y Letras por la Universidad de Alicante; He participado como ponente de congresos en Ecuador, España, Francia, Perú y Cuba; Asimismo, he publicado artículos de investigación sobre literatura hispanoamericana y didáctica de la literatura en revistas de Costa Rica, Venezuela, España, Cuba y Estados Unidos; Actualmente me desempeño como director y docente de la carrera de Educación Básica de la Universidad Nacional de Educación (UNAE), Ecuador. 


\title{
Artículo Original / Original Article
}

\author{
Edison Javier Padilla Padilla \\ e-mail: edison.padilla@unae.edu.ec
}

Nacido en Cuenca, Ecuador, el 30 de enero del año

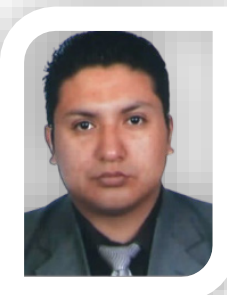
1983. Magíster en Docencia y Currículo para la Educación Superior por la Universidad Técnica de Ambato (UTA); y Magíster en Docencia de las Matemáticas por la Universidad de Cuenca (UCUENCA); la mayor parte de mi carrera la he desarrollado como docente de instituciones de nivel medio y universitario tanto en programas de pregrado como de posgrado; Actualmente me desempeño como docenteinvestigador en la Universidad Nacional de Educación (UNAE), Ecuador, en la cual aparte de la docencia universitaria, participó activamente en grupos de investigación e innovación educativa.

El contenido de este manuscrito se difunde bajo una Licencia de Creative Commons ReconocimientoNoComercial-Compartirlgual 4.0 Internacional 